\title{
Broadband and Compact 2-D Photonic Crystal Reflectors With Controllable Polarization Dependence
}

\author{
S. Boutami, B. Ben Bakir, H. Hattori, X. Letartre, J.-L. Leclercq, P. Rojo-Romeo, M. Garrigues, C. Seassal, and \\ P. Viktorovitch
}

\begin{abstract}
Two-dimensional (2-D) compact photonic crystal reflectors on suspended InP membranes were studied under normal incidence. We report the first experimental demonstration of 2-D broadband reflectors (experimental stopband superior to $200 \mathrm{~nm}$, theoretical stopband of $350 \mathrm{~nm}$ ). They are based on the coupling of free space waves with two slow Bloch modes of the crystal. Moreover, they present a very strong sensitivity of the polarization dependence, when modifying their geometry. A compact $\left(50 \times 50 \mu \mathrm{m}^{2}\right)$ demonstrator was realized and characterized, behaving either as a broadband reflector or as a broadband transmitter, depending on the polarization of the incident wave. Experimental results are in good agreement with numerical simulations.
\end{abstract}

Index Terms-Group velocity, InP membrane, photonic crystal (PC), photonic integrated circuits, polarization, waveguided modes.

\section{INTRODUCTION}

$\mathbf{P}$ HOTONIC crystals (PCs) have become an active research area for the development of integrated optics for various applications such as optical interconnects, telecommunications, sensor, imaging, etc. Most of the devices using PCs explore either their photonic bandgap (microcavities) or their in-plane guided modes (photonic integrated circuits). The drawback of using solely waveguided modes in PCs lies in the fact that they suffer from out-coupling losses due to diffractive coupling of waveguided optical modes with radiated modes into free space, when operating above the light line [1].

However, this out-coupling can be usefully exploited for the design of devices which are meant to operate above the light line, and not only in the waveguided regime. Instead of attempting to confine the light entirely into waveguide structures, the two-dimensional (2-D) PC can be deliberately opened to the third dimension of space by controlling the coupling between waveguided and radiated modes [2].

Very efficient reflectors can be obtained by using a single PC membrane. Subwavelength one-dimensional grating-based narrow-band and broadband mirrors were already demonstrated [3]-[6], but without considering the compactness of the device. Two-dimensional grating-based reflectors were demonstrated

Manuscript received September 22, 2005; revised December 9, 2005

The authors are with the Laboratoire d'Electronique Optoélectronique et Microsystèmes, Ecole Centrale de Lyon, 69134 Ecully, France (e-mail: salim.boutami@ec-lyon.fr; jean-louis.leclerq@ec-lyon.fr).

Digital Object Identifier 10.1109/LPT.2006.871833 with narrow-band characteristics [7], [8]. In this letter, we present the first experimental demonstration of 2-D compact $\left(50 \times 50 \mu \mathrm{m}^{2}\right)$ and broadband high-efficiency PC reflectors over a 350-nm wavelength range (200 $\mathrm{nm}$ experimentally, because of the limited spectral range of our analyzer). The broadband reflection was made possible by the exploitation of two modes of the PC. Moreover, we demonstrate the possibility offered by 2-D PCs to control accurately the polarization dependence of the reflection. In previous works, it was theoretically shown that a square lattice of rectangular air holes was able to provide a narrow-band polarization dependence (over $5 \mathrm{~nm}$ in the near infrared) [8]. In this work, we demonstrate that a slight deformation of circular air holes into elliptical ones in a square lattice leads to a very sensitive polarization dependence, over a $350-\mathrm{nm}$ wavelength range.

\section{General PrinciPle AND Design}

We consider a PC slab illuminated under an incident wave at a wavelength $\lambda$, and we consider also that a waveguided mode exists at this wavelength for the corresponding $k_{/ /}$-vector above the light line. Then, incident light will be coupled to the waveguided mode, and will be reemitted vertically by constructive interferences, because of the coupling from the waveguided mode to the radiated modes, with the corresponding constant time $\tau_{c}$. Thus, the PC slab behaves as a reflector.

For photonic integration, such reflectors should operate on a limited area. This can be achieved by confining photons, in other words, by trying to obtain a low group velocity for the waveguided mode [2]. Thus, photons will be able to couple to radiated modes before they laterally escape from the illuminated area. The time related to the escape of the photons from this area is noted $\tau_{g}$.

In order to make $\tau_{g}$ as large as possible, extremes of the dispersion characteristics, where the group velocity tends to zero, are very interesting. For a normal incidence, we are operating around the $\Gamma$ point. Around this point, a parabolic approximation may apply for the dispersion characteristics

$$
\omega=\omega_{0}+\frac{1}{2} \alpha(k)^{2}
$$

where $\alpha$ is the second derivative of the function $\omega(k)$ at $k=0$, that is the curvature of the graph around the $\Gamma$ point. 


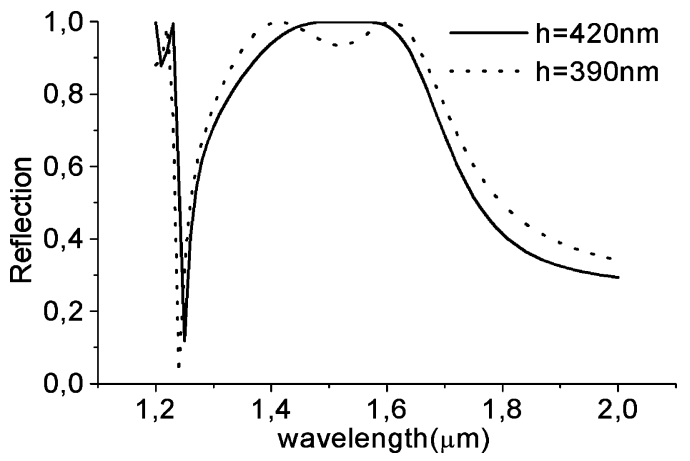

Fig. 1. Reflectivity of a square lattice of circular holes with a period of $1.2 \mu \mathrm{m}$, a filling factor of $70 \%$, for two InP membrane thicknesses, $h=420 \mathrm{~nm}$ (solid line) and $h=390 \mathrm{~nm}$ (dashed line). (RCWA simulations).

We can then deduce that the mean group velocity for a 2-D PC illuminated by a normal incidence beam of area $S$ is

$$
V_{g}=\frac{d \omega}{d k}=\alpha . k \cong \frac{\alpha}{\sqrt{S}} .
$$

The time related to the escape of the photons from the illuminated area is, therefore, given by [2], [9]

$$
\tau_{g} \cong \frac{S}{\alpha} \text {. }
$$

The PC structure can be designed in such a way as to minimize the curvature $\alpha$ around the $\Gamma$ point. Owing to the low mean group velocity, $\tau_{c} / \tau_{g}$ is made low enough for waveguided photons injected by the incident wave not to escape out of the illuminated area before being coupled back to the radiated modes. Such low-curvature bands can be obtained from high index contrast PCs. In the $1.3-1.55 \mu \mathrm{m}$ range, III-V semiconductors have great potential, especially for monolithic integration of active optical components. Therefore, we studied a PC membrane based on an InP-air periodic structuration, for which the index ratio is $3,17 / 1$.

We first considered a square lattice of circular air holes in the InP membrane. For a period of $1.2 \mu \mathrm{m}$, an air filling factor of $70 \%$, and a slab thickness of $420 \mathrm{~nm}$, rigorous coupled wave analysis (RCWA) [10] simulations show that we can obtain a highly reflective mirror over a large bandwidth (Fig. 1). The reflectivity is superior to $90 \%$ over a 250 -nm wavelength range. These reflection characteristics are essentially controlled by the presence of two resonant waveguided modes. The overlap between their resonances leads to a strong and broadband reflection. These two modes can be highlighted by slightly detuning the overlap. This can be done by modifying the filling factor, or even the thickness of the PC slab. For example, by reducing the thickness from 420 to $390 \mathrm{~nm}$, the overlap is not optimal anymore, and a small dip appears between the two maxima of reflection (dashed line in Fig. 1).

Because of the $90^{\circ}$ rotational symmetry of this structure, its optical response does not depend on the polarization of the incident exciting wave. However, by slightly varying the hole's shape, we can obtain polarization-sensitive reflectors. A square lattice of elliptical holes has been then designed in a 340-nmthick InP slab with a lattice period of $1.2 \mu \mathrm{m}$, and a short and long radius for elliptical holes of 500 and $550 \mathrm{~nm}$, respectively,

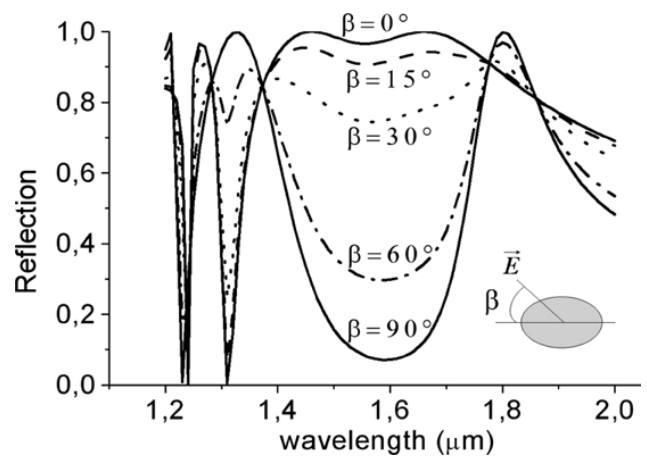

Fig. 2. Effect of the incident polarization on the reflectivity of a square lattice of elliptical holes (period $1.2 \mu \mathrm{m}$, long radius $550 \mathrm{~nm}$, short radius $500 \mathrm{~nm}$, membrane thickness $340 \mathrm{~nm}$ ) (RCWA simulations).

which corresponds to an ellipticity ratio of 1.1 (ratio of the long and short radii).

These parameters have been chosen in order to show a strong polarization sensitivity for a slight deformation of circular air holes, around $1.55 \mu \mathrm{m}$. Depending on the angle $\beta$ between the electric field of the incident wave and the longest axis of the ellipse, we gradually switch from a total reflection to a nearly total transmission on a very large bandwidth $(\Delta \lambda=350 \mathrm{~nm})$ centered around $\lambda=1.55 \mu \mathrm{m}$ (Fig. 2). For a polarization angle of $0^{\circ}$, we obtain a broadband reflection resulting from the overlap of two resonances. Then, by modifying gradually this angle from $0^{\circ}$ to $90^{\circ}$, we change the coupling time constants $\tau_{c 1}$ and $\tau_{c 2}$ between the two resonant modes of the slab and the radiated modes. The increase of these time constants results on the subsequent increase of the quality factors of the modes, and so to a deterioration of the overlap. Between the two resonances, the PC behaves as a transmitting membrane, because of its average index which corresponds to a half-wavelength thickness.

For higher ellipticity ratios, such polarization dependence properties can also be obtained, if adjusting the membrane thickness, lattice period, and long radius of elliptical air holes.

\section{EXPERIMENTAL RESULTS}

This polarization-dependent reflector was fabricated with an InP-InGaAs heterostructure. This was grown on InP substrate using low-pressure metal-organic vapor phase epitaxy. The epitaxial structure consists of a 775-nm half-wavelength-thick InGaAs sacrificial layer, that will be the air-gap once the device is fabricated, and a 340-nm-thick InP layer forming the PC suspended membrane. In one hand, the half-wavelength thickness of air in between is high enough to prevent from any significant evanescent coupling into the substrate. In the other hand, it results in the minimization of the influence of reflectivity at the substrate interface. The 2-D PC reflector fabrication is based on our photonic crystal-microoptoelectromechanical systems (PC-MOEMS) structures procedure; details can be found in [11]. Fig. 3 shows the scanning electron microscope (SEM) pictures of the $50 \times 50 \mu \mathrm{m}^{2}$ fabricated lattice of elliptical holes (the lowest and the highest radius of the ellipse are 500 and $550 \mathrm{~nm}$, respectively).

The reflectance of the structure was then characterized. For measurements, the incident beam from a monomode fiber was 

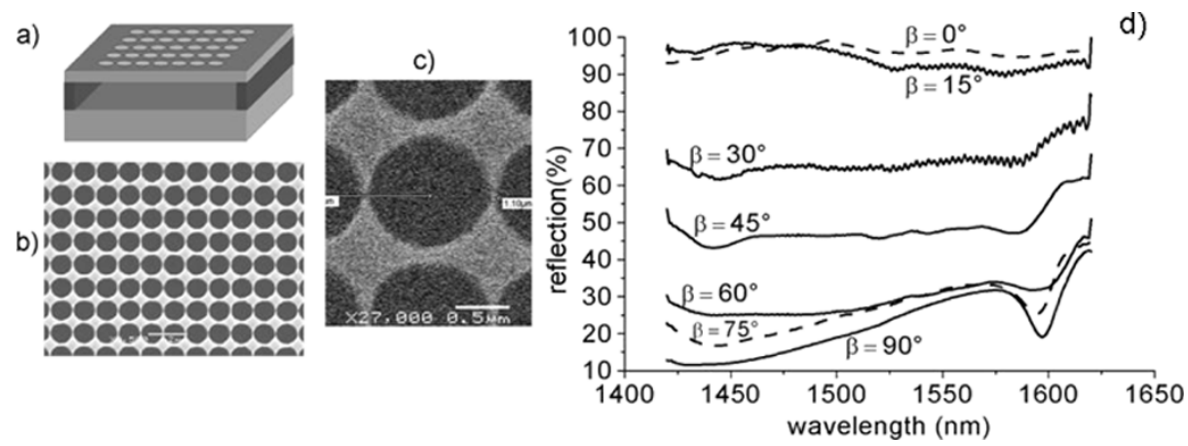

Fig. 3. (a) Schematic representation of the PC slab structure. (b) SEM general view. (c) SEM close-up view over one cell of the lattice. (d) Experimental reflectivity spectra of the square lattice of elliptical holes in the analyzer spectral range $(1.42-1.62 \mu \mathrm{m})$, for various angles of polarization of the incident wave.

coupled to the device using a lens collimator (waist diameter of $12 \mu \mathrm{m}$ ). The excitation light was provided by a large band light source combining superluminescent light-emitting diodes (SLEDs) and an erbium-doped fiber amplifier (EDFA). This source was connected to the collimator through a 50/50 directional coupler, followed by a polarizer and a section of polarization-maintaining fiber. The reflected beam was collected back by the collimator, separated on the directional coupler, and analyzed by a spectrum analyzer. The reference for $100 \%$ reflectance was taken onto a metal contact of the device under test. Fig. 3(d) shows the experimental reflectivity measurements between 1.42 and $1.62 \mu \mathrm{m}$ as a function of the polarization angle $\beta$. A good agreement was achieved between simulations and experimental results, which were independent of the location of the beam on the PC.

For polarization angles lower than $15^{\circ}$, the reflectivity is higher than $90 \%$ and the bandwidth of the reflector covers the whole measurement wavelength range $(200 \mathrm{~nm})$. When increasing the polarization angle from $30^{\circ}$ to $90^{\circ}$, the reflectivity is decreasing while a dip in the reflectance spectra is appearing progressively around $1.6 \mu \mathrm{m}$. This local dip of reflectivity is due to a resonant filter configuration when considering the air cavity located between the PC (top reflector) and the substrate (bottom reflector). Indeed, for high polarization angle, the reflectivity of the PC is on the same order of magnitude as the reflectivity at the interface of the substrate. Thus, the structure behaves as a Fabry-Pérot like transmitting resonant filter for the dip wavelength, which is around $1.6 \mu \mathrm{m}$.

\section{CONCLUSION}

In this letter, we presented a broadband and compact 2-D elliptical holes array-based PC reflector, with a sharp polarization dependence. This device is based on the interplay between radiated modes and two waveguided modes in the PC, which can be widely and finely adjusted by changing the geometrical parameters of the PC membrane (thickness, lattice period, and shape of holes). The experimental results are in good agreement with simulations.

This work opens the way to a wide variety of compact PC devices, in which properties of polarization dependence for specific applications can be accurately controlled by geometrical parameters of the PC.

This InP-based mirror has potential application when monolithically integrated in classical optoelectronic active and passive microdevices (filters, LEDs, VCSELs, photodetectors, etc.).

One can also imagine for example to obtain such polarization tunable/switchable reflectors by using a square lattice of circular air holes, filled with a birefringent material.

\section{ACKNOWLEDGMENT}

The authors would like to thank I. Sagnes and M. Strassner of Laboratoire de Photonique et de Nanostructures (Marcoussis, France) for help in providing the LP-MOCVD heterostructures.

\section{REFERENCES}

[1] M. Loncar, D. Nedeljkovic, T. P. Pearsall, J. Vukovic, A. Scherer, S. Kuchinsky, and D. C. Allan, "Experimental and theoretical confirmation of Bloch-mode light propagation in planar photonic crystal waveguides," Appl. Phys. Lett., vol. 80, no. 10, pp. 1689-1691, 2002.

[2] X. Letartre, J. Mouette, J. L. Leclercq, P. Rojo-Romeo, C. Seassal, and P. Viktorovitch, "Switching devices with spatial and spectral resolution combining photonic crystal and MOEMS structures," J. Lightw. Technol., vol. 21, no. 7, pp. 1691-1699, Jul. 2003.

[3] Z. S. Liu, S. Tibuleac, D. Shin, P. P. Young, and R. Magnusson, "Highefficiency guided-mode resonance filter," Opt. Lett., vol. 23, no. 19, pp. 1556-1558, Oct. 1998.

[4] Y. Ding and R. Magnusson, "Use of nondegenerate resonant leaky modes to fashion diverse optical spectra," Opt. Express, vol. 12, no. 9 , pp. 1885-1891, May 2004.

[5] C. F. R. Mateus, M. C. Y. Huang, Y. Deng, A. R. Neureuther, and C. J. Chang-Hasnain, "Ultrabroadband mirror using low-index cladded subwavelength grating," IEEE Photon. Technol. Lett., vol. 16, no. 2, pp. 518-520, Feb. 2004.

[6] C. F. R. Mateus, M. C. Y. Huang, L. Chen, C. J. Chang-Hasnain, and Y. Suzuki, "Broadband mirror (1.12-1.62 $\mu \mathrm{m})$ using single-layer subwavelength grating," IEEE Photon. Technol. Lett., vol. 16, no. 7, pp. 1676-1678, Jul. 2004.

[7] S. Peng and G. M. Morris, "Experimental demonstration of resonant anomalies in diffraction from two dimensional gratings," Opt. Lett., vol. 21, pp. 549-551, 1996.

[8] V. Lousse, W. Suh, O. Kilic, S. Kim, O. Solgaard, and S. Fan, "Angular and polarization properties of a photonic crystal slab mirror," Opt. Express, vol. 12, no. 8, pp. 1575-1582, Apr. 2004.

[9] X. Letartre, C. Monat, C. Seassal, and P. Viktorovitch, "An analytical and an experimental investigation of 2-D photonic crystal micro-lasers: Defect state (micro-cavity) versus band edge state (distributed feed-back) structures," J. Opt. Soc. Amer, vol. 22, pp. 2581-2595, Dec. 2005.

[10] M. G. Moharam and T. K. Gaylord, "Rigorous coupled-wave analysis of planar grating diffraction," J. Opt. Soc. Amer., vol. 71, pp. 811-818, 1981.

[11] J. L. Leclercq, P. Rojo-Romeo, C. Seassal, J. Mouette, X. Letartre, and P. Viktorovitch, "3D structuring of multilayer suspended membranes including 2-D photonic crystal structures," J. Vac. Sci. Technol. B, vol. 21, no. 6, pp. 2903-2906, Nov./Dec. 2003. 